\title{
Microbial Contamination and Antimicrobial Resistance Profiles Indicate Potential Risks of Infection at the Veterinary Medical Teaching Hospital - UFRGS, Porto Alegre, Brazil
}

\author{
Mariana Muller Giacon ${ }^{1}$, Franciele Maboni Siqueira² \& Amanda de Souza da Motta'
}

\begin{abstract}
Background: This study aimed to assess the level of bacterial contamination in the Small Animals Sector of the Veterinary Medical Teaching Hospital (HCV) of the Universidade Federal do Rio Grande do Sul (UFRGS). Firstly, a committee was invited to complete a questionnaire and to list critical sample sites for collection. With the identification of the places to be sampled, collections were made with sterile swabs on different surfaces of environments of the HCV. The identification of important bacteria in the veterinary area, in the different sampled environments, raises the concern for hygiene procedures in the veterinary hospital environment.
\end{abstract}

Materials, Methods \& Results: Sixteen samples were collected from these different areas, and microbiological analyses were performed. Standard counts of viable and strictly aerobic mesophilic microorganisms were realized. Collections were made to assess ambient air quality. With the microbiological analysis performed, bacteria of clinical importance were identified. To assess the resistance profile of the bacteria, the susceptibility test to antimicrobials was performed. MALDI-TOF/MS measurement identified 29 bacteria at the genus level and 10 bacteria at the species level and the antimicrobial susceptibility test was realized. Most of the isolates identified (60\%) were bacteria of the genus Staphylococcus spp. Regarding antimicrobial susceptibility analysis the 10 bacteria identified at the species level were assessed. Test results showed that the isolates S. aureus, S. epidermidis and S. haemolyticus - collected from treatment room 2 - and S. haemolyticus, which had been isolated from samples from treatment room 2 of the cattery, presented multiresistance. Pantoea ananatis isolates from room 5 also showed a multiresistant profile for erythromycin, cephalothin, vancomycin and ampicillin. Micrococcus luteus isolates from the $\mathrm{x}$-ray room and the kennel showed resistance to ceftazidime. Staphylococcus equorum isolates from room 4 were sensitive to all tested antimicrobials.

Discussion: In Brazilian legislation there are no official microbiological parameters for surfaces in a veterinary hospital environment. The microorganisms present in the air are transient and variable, and the number and types of airborne agents is determined by the various sources of contamination in the environment. These microorganisms can be found in suspension, particulate matter and water droplets. Veterinary medical care tables are potentially contaminated by the animals handling, including those that sometimes defecate or urinate during their medical visit. Frequent handwashing is also known to be an important means of personal protection and disease prevention, although it is estimated that only $40 \%$ of practitioners do so routinely. Based on these results, we recommend a plan of bacterial control and disinfection that should be implemented to ensure more effective sanitary conditions. Microorganism counts were high in some of the veterinary hospital environments tested, indicating that current disinfection and hygiene practices are not sufficient to control the establishment of these microorganisms at the study sites. In view of this, it is reasonable to conclude that permanent monitoring and assessment of the effectiveness of hygiene protocols is needed in different sectors of the hospital. This may be an essential tool in a preventive approach to stop the spread of selectively resistant microorganisms, as well as cases of hospital infections. In addition, continuous staff training and awareness of the importance of personal and environmental hygiene is vital for minimizing the presence of these microorganisms in hospitals and avoid their transmission to patients. Finally, a more systematic hygiene guideline should be implemented in areas that showed higher counts.

Keywords: antimicrobial resistance, cats, dogs, bacteria, hospital infection, veterinary hospital.

DOI: $10.22456 / 1679-9216.108992$ 


\section{INTRODUCTION}

Veterinary hospitals are similar to human hospitals in their unquestionable and considerable complexity. Thus, the increasing frequency of cases of hospital infections acquired by animals has obliged administrators, veterinarians and assistant staff to broaden their knowledge about this specific problem [15].

Infection control programs establish procedures aimed at preventing the spread of infections to patients, pet owners, veterinarians and other employees [6].

Routine practices should include effective and regular hand hygiene, the use of personal protective equipment, hospital cleaning and disinfection, and the appropriate management of environments and equipment. These procedures result in the removal of dirt, a reduction in microbial load and the elimination of multidrug-resistant strains. Nevertheless, it should be highlighted that, considering their purpose and how they are performed, these practices are clearly not intended to achieve a complete elimination of all microorganisms.

In Veterinary Medicine, this issue still hasn't been a subject of robust research, which makes any new study important for the implementation of more efficient preventive measures - such as the control, identification and measurement of the causes of infections - with a view towards reducing contamination. The present study aimed to assess the level of bacterial contamination in the Small Animals Sector of one Veterinary Medical Teaching Hospital (HCV). The data collected was to be later used in making decisions to lower the number of cases of infection as well as to improve planning for hospital health and safety guidelines.

\section{MATERIALS AND METHODS}

\section{Institution and data collection site}

The Veterinary Medical Teaching Hospital (HCV) of the Universidade Federal do Rio Grande do Sul (UFRGS), which receives an average of 20,000 patient visits per year, was the object of study of this research. It is a teaching hospital that is run by staff composed by Professors, DVM, residents, interns, students and other employees, that are directly in contact with animals and the facilities in which they are treated or kept. In recent years, the HCV-UFRGS has registered some cases of bacterial infections arising from or following admission to the hospital environment. Consequently, a Disinfection Commission was established in 2016 to plan and oversee the control of hospital infections as well as pest and rodent problems.

The study began with the completion of a questionnaire by 4 members of the Disinfection Commission (three veterinarians and one collaborator) through which they were asked to assess the hygienic and sanitary status of the VH. They should also identify the most critical contamination sites, based on the history of infectious diseases available in Sector of Medical Archives (SMA), animal transit within the institution and the appearance of facilities in terms of hygiene. After analysing their answers, specific sites were chosen for being more critical for sample collection, in order to assess the level of environmental microbial contamination.

\section{Sampling procedures for microbiological analysis}

Environmental samples were taken from surfaces and air sedimentation. For surfaces, sterile swabs were moistened in $0.1 \%$ peptone water ${ }^{1}$ and rubbed against them three times in different directions, within an area of $100 \mathrm{~cm}^{2}$. The collected swabs were packed in $9 \mathrm{~mL}$ of $0.1 \%$ sterile peptone water ${ }^{1}$, refrigerated and transported to the laboratory. As for the sedimentation of air, Plate Count Agar (PCA) ${ }^{2}$ plates were used. These were left in each room for 20 min of direct exposure, then collected and sent to the laboratory.

Standard counting of viable strict and non-strict aerobic mesophilic microorganisms and collection by spontaneous sedimentation

For the plate count of surface swabs, we used the spread-plate technique by surface seeding $0.1 \mathrm{~mL}$ of each dilution (10-1 to 10-5) and spreading it evenly over the middle surface with a Drigalski handle. Plates were incubated at $36^{\circ} \mathrm{C}$ for $48 \mathrm{~h}$. For the evaluation of the microbiological quality of air by spontaneous sedimentation, plates were incubated at $36^{\circ} \mathrm{C}$ for 48 h. For reading, all $\mathrm{PCA}^{2}$ plates containing between 25 and 250 colonies were selected for counting. Results of samples collected by swabbing were expressed in colony forming units per square centimetre $\left(\mathrm{CFU} / \mathrm{cm}^{2}\right)$, whereas samples of air sedimentation were expressed in CFU per plate (CFU/plate).

Selection and identification of isolates via Matrix-Associated Laser Desorption/Ionization Time-Of-Flight Mass Spectrometry (MALDI-TOF/MS)

The criterion for bacteria selection was the presence of different colonial morphotypes on the 
same culture plate; all morphotypes observed on each plate were used for the subsequent analyses. Selected colonies were seeded on Tryptic Soy Agar (TSA) ${ }^{1}$ and incubated at $36^{\circ} \mathrm{C}$ for $24 \mathrm{~h}$. From this growth, one colony of each isolate was resuspended in $300 \mu \mathrm{L}$ of ultrapure water and $900 \mu \mathrm{L}$ of absolute ethanol. Initially, the samples were centrifuged and the supernatant discarded. After, $70 \%$ formic acid was added to the pellet and a new centrifugation step was performed. Subsequently, $1 \mu \mathrm{L}$ of supernatant was pipetted onto a stainless steel plate and allowed to dry at room temperature. Subsequently $1 \mu \mathrm{L}$ of the matrix was added. The analyses were performed with the MALDI Biotyper 4.0, MBT OC software ${ }^{3}$.

\section{Antimicrobial susceptibility test}

For this test, the agar disk diffusion method was adopted, in accordance with the recommendations of the Clinical and Laboratory Standards Institute [3]. The assays were performed with cultures of the TSA ${ }^{1}$ agar isolates obtained after incubation at $36^{\circ} \mathrm{C}$ for 24 h. From this growth, bacterial suspensions matching the turbidity of a McFarland 0.5 standard were seeded on the surface of plates containing Mueller-Hinton agar $^{1}$. The tested antibiotics were: Ceftazidime $30 \mu \mathrm{g}$ $\left(\right.$ CAZ 30) ${ }^{4}$, Tetracycline $30 \mu \mathrm{g}$ (TET 30) ${ }^{4}$, Amikacin $30 \mu \mathrm{g}$ (AMI 30) ${ }^{4}$, Cephalothin $30 \mu \mathrm{g}$ (CFL 30) ${ }^{4}$, Vancomycin $30 \mu \mathrm{g}(\mathrm{VAN} 30)^{4}$, Imipenem $10 \mu \mathrm{g}$ (IPM 10) ${ }^{4}$, Chloramphenicol $30 \mu \mathrm{g}(\mathrm{CLO} 30)^{4}$, Ampicillin $10 \mu \mathrm{g}$ (AMP 10) ${ }^{4}$, Meropenem $10 \mu \mathrm{g}$ (MER 10) $)^{4}$ Plates were incubated at $36^{\circ} \mathrm{C}$ for $18 \mathrm{~h}$, followed by the interpretation of the diameters of inhibition halos.

\section{RESULTS}

During the visit to the HCV it was noted that some hospital sectors have a greater volume of movement of employees, veterinarians, tutors and animals, such as the hospitalization sectors and the kennel treatment room. Hygiene procedures on the surfaces of tables in these places were done the correct way. However, in other sectors, no disinfection of surfaces after use was observed, except in service room 3. In service room 1 of the cattery, staff reported the execution of hygiene protocols on the examination table after each patient.

Positive points were identified through the field visit, in addition to the analysis of the questionnaire answers, namely: i) existence of the Disinfection Commission, ii) training of hospital staff; and iii) the standardiza- tion of products used for the cleaning and disinfection of all sectors of the HCV. Nevertheless, some negative points were also identified, such as: i) inconsistent periodicity of training, ii) difficulty in checking cleaning practices, iii) inconsistent maintenance of good hygiene practices and iv) a failure to carry out hygiene protocols in some environments after use. According to the responses to the questionnaire, these points are also considered important challenges by the Disinfection Commission.

After this analysis, we chose 16 sites for sample collection on the surfaces of various VH sectors. Facilities with higher numbers of patient visits and more intense animal transit were taken into consideration. In general, the VH was considered to be under good hygienic-sanitary conditions at the time of data collection (Table 1).

We have performed the mesophilic microorganisms count, which provide data that the $\mathrm{VH}$ is an important environmental reservoir of pathogens. There were no patients being attended in the treatment rooms 1 (SA1M), 2 (SA2M) at the time of data collection. In both these spaces, the examination table (DIM) and cage (DIGa) of the isolation room (DI), the cattery cage (GATGa) and the examination table of the x-ray (RXM), were all visually clean and sanitised. In Table 1 we can see that in SA1M and SA2M the counts from surface samples were higher while air sedimentation counts were within acceptable ranges, in accordance with the American Public Health Association [1]. These results show that an absence of animals and people in a particular area is associated with lower levels of microbial growth. On the other hand, it is also possible to conclude that cleaning practices on surfaces in these rooms were not performed satisfactorily. This is a different finding from those of other areas of which surface and sedimentation sample counts registered within acceptable standards. In treatment room 3 (SA3), and in a service room 1 of the cattery (GATSA1), the hygiene of the examination tables (SA3M) had been performed by the veterinarian after the last visit, and samples from these places revealed low counts (Table 1).

The examination tables of rooms 4 and 5 (SA4M and SA5M), and service room 2 of the cattery (GATSA2), as well as in the general kennel and cattery sections, sample collection was carried out while the veterinarians were attending animal patients. Nevertheless, the kennel (CANM) and cattery (GATMT) examination tables were not in use at the time of sample collec- 
tion. The cleaning staff had sanitised these local after the last patient. While air sedimentation counts were high, surface scores were not; this data demonstrates that although the disinfection of surfaces was satisfactory, the ongoing patient visits that were underway in those environments could have contributed to the microbial growth gauged via sedimentation (Table 1). The examining tables of the ultrasound (US) and x-ray (RXM) rooms were not in use, but these had not been sanitised before sample collection. Thus, high counts were observed: $17.83 \mathrm{CFU} / \mathrm{cm}^{2}$ and $43.59 \mathrm{CFU} / \mathrm{cm}^{2}$, respectively. One can see that even surfaces which had been reported as sanitised presented microbial growth, even if most still remained within the standard of up to $2 \mathrm{CFU} / \mathrm{cm}^{2}[1]$. This fact may suggest that the products used for hygiene, as well as the adopted procedures, are proving to be efficient. In the analyses of air quality was observed high bacterial growth as observed in Table 1, except in the cattery facilities.

From the isolation of bacterial colonies with different morphotypes, 55 isolates were selected for identification. Of the 55 analysed isolates, 46 were identified and 10 of these were further precisely identified at the species level (Table 2), as well as one type of yeast. The other 29 were identified at the gender level and the remaining six were impossible to identify more precisely according to MALDI-TOF criteria.

In general, most of the isolates identified (60\%) were bacteria of the genus Staphylococcus spp. Regarding antimicrobial susceptibility analysis the 10 bacteria identified at the species level were assessed. Test results showed that the isolates $S$. aureus, S. epidermidis and S. haemolyticus - collected from treatment room 2 - and S. haemolyticus, which had been isolated from samples from treatment room 2 of the cattery, presented multiresistance. Pantoea ananatis isolates from room 5 also showed a multiresistant profile for erythromycin, cephalothin, vancomycin and ampicillin. Micrococcus luteus isolates from the x-ray room and the kennel showed resistance to ceftazidime. Staphylococcus equorum isolates from room 4 were sensitive to all tested antimicrobials.

Table 1. Selected sample collection points at the Veterinary Medical Teaching Hospital (HCV) of the Universidade Federal do Rio Grande do Sul (UFRGS) and results of the standard count of strict and non-strict aerobic mesophilic microorganisms via surface swabs and spontaneous air sedimentation expressed in $\mathrm{CFU} / \mathrm{cm}^{2}$ and $\mathrm{CFU} /$ plate.

\begin{tabular}{|c|c|c|c|}
\hline Sample site code & Facility/object of sample collection & Surface Swabs & Spontaneous air sedimentation \\
\hline SA1M & Table Room 1 & $4 \times 10^{2}$ & 17.83 \\
\hline SA2M & Table Room 2 & $3.5 \times 10^{3}$ & 7.92 \\
\hline SA3M & Table Room 3 & 0 & 9.90 \\
\hline SA5M & Table Room 5 & $<25^{*}$ & 99.07 \\
\hline DIM & Infectious disease room: treatment table & 0 & 11.88 \\
\hline $\mathrm{DIGa}$ & Cage for hospitalization of animals with infectious diseases & 0 & - \\
\hline RXM & X-ray room examination table & $<25^{*}$ & 43.59 \\
\hline RXA & Apron used in the $\mathrm{X}$-ray room & $<25^{*}$ & - \\
\hline US & Ultrasound room examination table & 0 & 17.83 \\
\hline CANM & Kennel treatment table & 0 & 63.41 \\
\hline Cane & Kennel for hospitalization & $<25^{*}$ & - \\
\hline GATSA1 & Examination table of room 1 of the cattery & 0 & 5.94 \\
\hline GATSA2 & Examination table of room 2 of the cattery & $<25^{*}$ & 5.94 \\
\hline GATMT & Treatment table of the cattery & $<25^{*}$ & 0 \\
\hline Cat & Cage for hospitalization at the cattery & 0 & - \\
\hline
\end{tabular}

*<25: growth less than 25 CFUs. (-) Collection points which were not sampled. 
M.M. Giacon, F.M. Siqueira \& A.S. Motta. 2021. Microbial Contamination and Antimicrobial Resistance Profiles Indicate Potential Risks of Infection at the Veterinary Medical Teaching Hospital... Acta Scientiae Veterinariae. 49: 1800.

Table 2. Identification of isolates at species-level by MALDI-TOF/MS.

\begin{tabular}{cc}
\hline Site and Collection Method & Microorganism Identification \\
\hline Surface SA1M-1 & Staphylococcus haemolyticus \\
Surface SA2M-3 & Staphylococcus aureus \\
Surface SA2M-4 & Staphylococcus epidermidis \\
SA2-4 sedimentation & Staphylococcus epidermidis \\
SA4-1 sedimentation & Staphylococcus equorum \\
SA5-1 sedimentation & Pantoea ananatis \\
RXM-2 surface swab & Micrococcus luteus \\
CAN-2 sedimentation & Micrococcus luteus \\
GATSA1-2 sedimentation & Leclercia adecarboxylata \\
GATSA2-3 surface swab & Staphylococcus haemolyticus
\end{tabular}

Database analysis performed by Biotyper 4.0 software MBT OC.

\section{DISCUSSION}

Regarding the analyses of the microbiological quality of the air rooms where there was greater circulation of people and animals, the growth of bacteria by sedimentation of the air was greater, except in the cattery facilities (Table 1). This data shows that microorganisms circulate in greater numbers where there is greater volume of human and animal transit. As recommendation for simple sedimentation plates is up to $30 \mathrm{CFU} / \mathrm{cm}^{2}$, the results obtained in SA5M and in CANM are outside of acceptable standards [1].

It is important to note that in Brazilian legislation there are no official microbiological parameters for surfaces in a veterinary hospital environment. However, there are regulations for the microbiological quality of air in other environments, which forces institutions to adopt Resolution No. 9 as a guide for air quality studies [2]. The microorganisms present in the air are transient and variable, and the number and types of airborne agents is determined by the various sources of contamination in the environment. These microorganisms can be found in suspension, particulate matter and water droplets. Veterinary medical care tables are potentially contaminated by the animals handling, including those that sometimes defecate or urinate during their medical visit. Frequent handwashing is also known to be an important means of personal protection and disease prevention, although it is estimated that only $40 \%$ of practitioners do so routinely

Staphylococci are Gram-positive bacteria, being the higher abundant bacterial taxon in the skin microbiota. Staphylococcus spp. are resistant to variations in $\mathrm{pH}$ and desiccation, especially in exudates, and may remain present for weeks in a given environment, which could explain the high identification level of this genera in the present study [14].

The skin microbiota of pets is highly complex with large inter-individual variability and differences among skin sites [4]. Environmental variations could provide populational instability allowed important disease, such as folliculitis, otitis, pyoderma, opportunistic infections, urinary tract infections, impetigo and endocarditis.

Veterinary nosocomial infections have been recognised as increasing in frequency and, as in human medicine, the most common pathogens are Staphylococcus spp., Enterococcus spp., members of the Enterobacteriaceae family, Clostridium difficile, Acinetobacter spp. and Pseudomonas spp. [12].

Regarding antimicrobial susceptibility analysis the 10 bacteria identified at the species level were assessed. Test results showed that the isolates $S$. aureus, S. epidermidis and S. haemolyticus - collected from treatment room 2 - and S. haemolyticus, which had been isolated from samples from treatment room 2 of the cattery, presented multiresistance. That is to say resistance to 3 or more antimicrobial categories [10]. Pantoea ananatis isolates from room 5 also showed a multiresistant profile for erythromycin, cephalothin, vancomycin and ampicillin. To date, there have been no reports in the literature about $P$. ananatis being resistant to vancomycin; yet nosocomial infections in animals caused by Enterococcus spp. resistant to vancomycin, as well as the identification of animal carriers have already been described [7]. The detection of these bacteria resistant to this drug in veterinary hospitals is very worrisome and serves as a warning about the risks 
to public health, since species such as $P$. ananatis may play an important role in the maintenance and spreading of factors which provide resistance to vancomycin. Micrococcus luteus isolates from the X-ray room and kennel showed resistance to ceftazidime. Staphylococcus equorum isolates from room 4 were sensitive to all tested antimicrobials.

These results revealed that the resistance frequency is higher in isolates of the genus Staphylococcus spp. The multiresistance of Staphylococcus is extremely important, both from a clinical and microbiological point of view, since bacterial resistance causes difficulties in the treatment of animals and multiresistant bacteria can spread to the wider environment [13]. There was an even greater prevalence of resistance to erythromycin (44\%), tetracycline (33\%), the ampicillin $(55 \%)$ and ceftazidime $(66 \%)$. The presence of the Staphylococcus spp. in different hospital environments is another point of concern, since it shows that these microorganisms have become persistent in these environments. Moreover, their antimicrobial resistance profiles demonstrate the difficulty to be faced in reducing their numbers [14].

It is important to highlight that in this study resistant bacteria were isolated from the environment and not from animals under treatment. This is a compelling observation because it draws attention to the movement of environmental bacteria with multidrug resistance patterns. In turn, this raises the alarm against the indiscriminate use of antimicrobials, which allows for the natural selection of resistant bacteria. Furthermore, the abuse and indiscriminate use of antimicrobial agents in human and veterinary clinical practice, especially in hospital settings, promote the natural selection of antibiotic resistant strains, such as Staphylococcus spp. S. aureus and S. pseudointermedius in particular are extremely versatile in developing resistance to antimicrobial agents. This supports their survival in hospital settings and its diffusion among patients [11]. The multiresistant profile that was observed in some isolates becomes even more alarming when antibiotics such as ceftazidime fail to be effective, since it is a third generation antimicrobial, highlighting the increase of more scarce therapeutic options [5,8].

The consequences of inappropriate antimicrobial use in small animals do not differ from those in human medicine: the quantities and standards of use determine the rate of appearance of resistant strains. Several retrospective studies have reported an increased prevalence of resistance in different bacteria isolates from companion animals $[7,12,15]$. In London, Methicillin-resistant Staphylococcus aureus (MRSA) was detected in $17.9 \%$ of the employees of a veterinary hospital and in $9 \%$ of hospitalised dogs; these samples were resistant and related to isolates from human hospitals [9]. In Germany, 869 samples from small animals at a veterinary medical school were studied and MRSA was detected in 18 dogs, four cats, a guinea pig, a rabbit, an aquatic turtle and a bat [16].

Microorganism counts were high in some of the veterinary hospital environments tested, indicating that current disinfection and hygiene practices are not sufficient to control the establishment of these microorganisms at the study sites. In view of this, it is reasonable to conclude that permanent monitoring and assessment of the effectiveness of hygiene protocols is needed in different sectors of the hospital. This may be an essential tool in a preventive approach to stop the spread of selectively resistant microorganisms, as well as cases of hospital infections. In addition, continuous staff training and awareness of the importance of personal and environmental hygiene is vital for minimizing the presence of these microorganisms in hospitals and avoid their transmission to patients. Finally, a more systematic hygiene guideline should be implemented in areas that showed higher counts.

\section{CONCLUSION}

With this work it was possible to identify mandatory and important points of contamination within one Veterinary Medical Teaching Hospital. Staphylococcus spp. were isolated in various environments and the resistance profile was observed. The existence of a Standing Committee for infection control is necessary in Veterinary Hospitals in order to stablish guidelines and recommendations according to each particular situation.

\section{MANUFACTURERS}

${ }^{1}$ Oxoid Ltd. Basingstoke, Hampshire, UK.

${ }^{2}$ HiMedia Laboratories. Mumbai, India.

${ }^{3}$ Bruker Corporation. Billerica, MA, USA.

${ }^{4}$ Laborclin Produtos para Laboratório Ltda. Pinhais, PR, Brazil.

Acknowledgements. The research team would like to thank the Veterinary Hospital of Universidade Federal do Rio Grande do Sul, for allowing us to carry out our study at their institution.

Declaration of interest. The authors declare no conflicts of interest. The authors alone are responsible for the content and writing of paper. 


\section{REFERENCES}

1 American Public Health Association. 1998. Standard methods for the examination of water and wastewater. 20th edn. New York: APHA/AWWA.

2 Brasil. Agência Nacional de Vigilância Sanitária. 2003. Orientação Técnica Sobre Padrões Referenciais de Qualidade do Ar Interior em Ambientes Climatizados Artificialmente de Uso Público e Coletivos. Resolução N.9 de 16 de Janeiro de 2003. Brasília: Diário Oficial da União, 10p.

3 Clinical and Laboratory Standards Institute (CLSI). 2016. Performance Standards for Antimicrobial Susceptibility Testing M100-S26. 26th edn. Wayne: CLSI, 256p.

4 Cuscó A., Belanger J.M., Gershony L., Islas-Trejo A., Levy K., Medrano J.F., Sánchez A., Oberbauer A.M. \& Francino O. 2017. Individual signatures and environmental factors shape skin microbiota in healthy dogs. Microbiome. 5(1): 139-153.

5 Fitzgerald J.R. 2009. The Staphylococcus intermedius group of bacterial pathogens: species reclassification, pathogenesis and the emergence of meticillin resistance. Veterinary Dermatology. 20(5-6): 490-495.

6 Guardabassi L. 2012. Veterinary hospital-acquired infections: The challenge of MRSA and other multidrug-resistant bacterial infections in veterinary medicine. The Veterinary Journal. 193(2): 307-308.

7 Hamilton E., Kruger J.M., Schall W., Beal M., Manning S.D. \& Kaneene J.B. 2013. Acquisition and persistence of antimicrobial resistant bacteria isolated from dogs and cats admitted to a veterinary teaching hospital. Journal of the American Veterinary Medical Association. 243(7): 990-1000.

8 Hanselman B.A., Kruth S.A., Rousseau J., Low D.E., Willey B.M., McGeer A. \& Weese J. 2006. Methicillin-resistant Staphylococcus aureus colonization in veterinary personnel. Emerging Infectious Disease. 12(12): 1933-1938.

9 Loeffler A., Boag A.K., Sung J., Lindsay J.A., Guardabassi L., Dalsgaard A., Smith H., Stevens K.B. \& Lloyd D.H. 2005. Prevalence of methicillin-resistant Staphylococcus aureus among staff and pets in a small animal referral hospital in the UK. Journal of Antimicrobial and Chemotherapy. 56(4): 692-697.

10 Magiorakos A.P., Srinivasan A., Carey R.B., Carmeli Y., Falagas M.E., Giske C.G., Harbarth S., Hindler J.F., Kahlmeter G., Olsson-Liljequist B., Paterson D.L., Rice L.B., Stelling J., Struelens M.J., Vatopoulos A., Weber J.T. \& Monnet D.L. 2012. Multidrug-resistant, extensively drug-resistant and pandrug-resistant bacteria: an international expert proposal for interim standard definitions for acquired resistance. Clinical Microbiology and Infection. 18(3): 268-281.

11 Mendes C., Oplustil C., Sakagami E., Turner P. \& Kiffer C. 2005. Antimicrobial susceptibility in intensive care units: MYSTIC Program Brazil 2002. The Brazilian Journal of Infectious Disease. 9(1): 44-51.

12 Murphy C.P., Reid-Smith R.J., Boerlin P., Weese J.S., Prescott J.F., Janecko N., Hassard L. \& McEwan S.A. 2010. Escherichia coli and selected veterinary and zoonotic pathogens isolated from environmental sites in companion animal veterinary hospitals in southern Ontario. The Canadian Veterinary Journal. 51(9): 963-972.

13 Santos L.R, Scalco Neto J.F., Rizzo N.N., Bastiani P.V., Rodrigues L.B., Ferreira D., Schwants N., Barcellos H.H.A. \& Brun M.V. 2007. Cleaning, disinfection, and biosecurity procedures at Universidade de Passo Fundo Veterinary Hospital (HV-UPF). Acta Scientiae Veterinariae. 35(3): 357-362.

14 Santos L.R., Scalco Neto J.F., Rizzo N.N., Bastiani P.V., Rodrigues L.B., Barcellos H.H.A. \& Brun M.V. 2010. Environmental contamination of veterinary hospital and profile of antimicrobial susceptibility of isolated bacteria. Ciência Animal Brasileira. 11(2): 384-389.

15 Stull J.W. \& Weese J.S. 2015. Hospital-associated infections in Small Animal Practice. Veterinary Clinics of North America: Small Animal Practice. 45(2): 217-233.

16 Walther B., Wieler L.H., Friedrich A.W., Hanssen A., Kohn B., Brunnberg L., Lübke-Becker A. 2008. Methicillinresistant Staphylococcus aureus (MRSA) isolated from small and exotic animals at a university hospital during routine microbiological examinations. Veterinary Microbiology. 127(1-2):171-178. 